\title{
APLICAÇÃO DE NANOPARTÍCULAS DE FERRO ZERO-VALENTE (nFeZ) NA REMEDIAÇÃO DE SOLOS E ÁGUAS SUBTERRÂNEAS CONTAMINADAS: UMA REVISÃO
}

\author{
Sidiane Manfron ${ }^{\mathrm{a}, *,(1)}$, Antonio Thoméa, Iziquiel Cecchim ${ }^{\mathrm{a}}$ e Krishna R. Reddy \\ aDepartamento de Engenharia Civil e Ambiental, Universidade de Passo Fundo, 99001-970 Passo Fundo - RS, Brasil \\ bepartment of Civil and Materials Engineering, University of Illinois at Chicago, 842 West Taylor St., Chicago, IL 60607, USA
}

Recebido em 05/12/2019; aceito em 05/02/2020; publicado na web em 14/04/2020

\begin{abstract}
APPLICATION OF ZERO-VALENT IRON NANOPARTICLES (nZVI) ON THE REMEDIATION OF CONTAMINATED SOIL AND GROUNDWATER: A REVIEW. The remediation of contaminated soil and water is a relevant topic, being the focus of intense research. The use of nanoparticles to degrade contaminants has been consolidated as a sustainable remediation technology, with promising results worldwide. In this scenario, the zero-valent iron nanoparticles (nZVI) which, due to their properties, prove to be an important element to be used in remediation techniques, in addition to being efficient and low cost. In this article the objective is to present a bibliographic review on nanotechnology applied to soil and groundwater remediation, especially the application of nZVI, considering its main characteristics, reactivity performance, pollutants that can be degraded, application and transport techniques and main advantages and disadvantages. Issues related to toxicity and factors related to in situ applications are also presented. It is considered the results that have been observed, there is feasibility for the research to intensify even more, due to the efficiency of this nanomaterial on pollutants present in soils and waters, and it is important to expand the studies in Brazilian soils, since most of the research is international, and it is not possible to directly transfer knowledge acquired from temperate soils to residual soils of tropical and subtropical climate.
\end{abstract}

Keywords: nanoremediation; zero-valent iron (nZVI); toxicity mechanisms; transport.

\section{INTRODUÇÃO}

Solos e águas subterrâneas contaminadas podem ser conceituados como locais que apresentam processos, de origem natural ou antrópica, caracterizados pela presença de substâncias químicas em concentrações capazes de causar agravos à saúde humana e ao meio ambiente. A discussão acerca da contaminação do ambiente exige um olhar sobre os elementos potencialmente prejudiciais e que causam efeitos sobre os recursos naturais e a saúde da população, sendo que os processos industriais, a mineração, a agricultura intensiva e o descarte incorreto de resíduos fazem parte das principais atividades que geram contaminação do solo e água. ${ }^{1}$

Nos Estados Unidos a preocupação com as áreas contaminadas tem ampliado a atuação da Agência de Proteção Ambiental (EPAUSA). A existência de milhares de locais contaminados decorrentes de inúmeras atividades industriais, agrícolas ou de mineração, despertou para a necessidade de rastrear, identificar, monitorar e gerir programas de descontaminação, uma vez que além da questão ambiental é grande o impacto econômico gerado ao país. ${ }^{2} \mathrm{Na}$ Europa, estimam-se 2,5 milhões de locais onde ocorreram ou ocorrem atividades poluidoras, sendo que 65.500 locais foram remediados com predominância de óleos minerais e metais pesados como principais contaminantes. Os custos médios anuais envolvidos para avaliação e descontaminação representam cerca de 4,3 bilhões de euros, onde, mais de $32 \%$ das despesas totais provêm do orçamento público. ${ }^{3}$

No Brasil, atualmente é desconhecido o número total de áreas contaminadas. Em maio de 2002, a Companhia de Tecnologia de Saneamento Ambiental de São Paulo (CETESB) divulgou pela primeira vez a lista de áreas contaminadas no estado de São Paulo, destacando a existência de 255 áreas contaminadas. Esse registro vem sendo constantemente atualizado e, em 2018, atingiu o número de 897 áreas contaminada com risco confirmado, 697

*e-mail: sidianemanfron@gmail.com áreas contaminadas sob investigação e 1.441 áreas em processo de remediação. ${ }^{4}$

Atualmente, há tecnologias disponíveis para remediação de solos e águas subterrâneas contaminados, podendo ser aplicadas ex situ e in situ, sendo que estas últimas estão ocupando cada vez mais espaço no mercado, devido às grandes vantagens técnicas e econômicas que apresentam..$^{5,6}$

Nesse contexto, a tecnologia em nanoescala tem sido considerada representativa tendo em vista o impacto positivo que o uso de materiais nanoestruturados pode trazer para diversas áreas, incluindo a preservação do meio ambiente. ${ }^{7}$ A aplicação de nanotecnologia gera benefícios, uma vez que pode atuar na prevenção de poluição ou dos danos indiretos, contribuindo no tratamento ou remediação, tendo em vista que os nanomateriais apresentam propriedades de adsorção de metais e substâncias orgânicas que podem ser utilizados também para detecção e monitoramento de poluição. ${ }^{8}$

As tecnologias de remediação que utilizam nanopartículas podem ser divididas em dois grupos com base na reação química envolvida. $\mathrm{O}$ primeiro grupo diz respeito às tecnologias redutivas que usam as nanopartículas como doadoras de elétrons para transformar ou converter o contaminante em uma forma menos tóxica e menos móvel. A segunda é a tecnologia de estabilização e sorção, usando nanopartículas como um agente de sorbente, coprecipitante ou imobilizante do contaminante. ${ }^{9}$

Há evidências comprovadas sobre a efetividade e eficácia da aplicação da nanotecnologia em processos de remediação de solos e águas subterrâneas contaminadas por metais pesados, contaminantes inorgânicos, orgânicos e emergentes, sendo que se tem observado o uso predominante de nanopartículas à base de ferro. ${ }^{10,11}$

As nanopartículas de ferro na valência zero (nFeZ) agem como agentes redutores de compostos orgânicos e inorgânicos. ${ }^{12}$ Essas partículas podem variar de 10 a $100 \mathrm{~nm}$ de diâmetro, permitindo um maior acesso aos contaminantes. ${ }^{13}$ Sua principal característica é o alto potencial biocida. Além disso, após a oxidação, influencia 
os micro-organismos estimulando a atividade microbiológica pela biodisponibilidade do ferro oxidado, ${ }^{14}$ sendo por isso, considerado muito reativo e importante agente redutor de poluentes. ${ }^{15}$

Por ser um material versátil, o $\mathrm{nFeZ}$ apresenta representatividade na engenharia ambiental, podendo atuar como remediador em grande variedade de contaminantes redutíveis, incluindo os compostos organoclorados e outros metais pesados, de forma relativamente barata. A aplicabilidade prática das partículas de nanoferro reside no fato de gerar estados de oxidação e redução, sendo o ferro metálico um efetivo doador de elétrons. ${ }^{16}$

Nesse sentido, buscando apresentar uma revisão bibliográfica acerca do tema, o presente estudo traz elementos teóricos sobre o uso do $\mathrm{nFeZ}$ na remediação de solos e águas contaminadas, considerando as principais características e performance do $\mathrm{nFeZ}$ nos processos, tipos de poluente que podem ser remediados, vantagens e viabilidade do uso dessa tecnologia.

\section{USO DO FERRO ZERO-VALENTE (nFeZ) EM PROCESSOS DE REMEDIAÇÃO}

Os primeiros trabalhos usando nanopartículas metálicas para descontaminação de águas subterrâneas contaminadas com organoclorados foram realizados na década de 1990. Wang e Zhang ${ }^{17}$ sintetizaram em laboratório, em escala nano, partículas bimetálicas $(\mathrm{Pd} / \mathrm{Fe}, \mathrm{Pd} / \mathrm{Zn}, \mathrm{Pt} / \mathrm{Fe}, \mathrm{Ni} / \mathrm{Fe})$ e as aplicaram através de teste de bancada para remediação de diversos poluentes clorados e alguns organoclorados aromáticos. Neste estudo verificou-se que ocorria uma rápida e completa degradação dos poluentes pelo uso das nanopartículas, observando-se, pela primeira vez, que dentro dos elementos bimetálicos estudados os metais Fe e $\mathrm{Zn}$ serviam primeiramente como doadores de elétrons, enquanto os metais Pd, Ni e Pt como catalisadores.

A partir de então, a pesquisa com as nanopartículas e a utilização dessa tecnologia nos processos de remediação de contaminantes cresceu, sendo que Ponder et al. ${ }^{18}$ foram os primeiros a utilizarem unicamente as nanopartículas de ferro na valência zero ( $\mathrm{nFeZ}$ ) para descontaminação de água subterrânea contendo contaminantes metálicos, em escala de bancada. Os contaminantes avaliados pelos autores foram o Cromo (Cr (VI)) e o Chumbo ( $\mathrm{Pb}$ (II)) e verificaram que o uso de $\mathrm{nFeZ}$ rapidamente transformava o $\mathrm{Cr}$ (VI) em $\mathrm{Cr}$ (III) e o $\mathrm{Pb}$ (II) em $\mathrm{Pb}(0)$, considerando o material adequado para o uso em remediação in situ.

Apesar de diferentes materiais em nano escala serem estudados, a aplicação da tecnologia contendo $\mathrm{nFeZ}$ tem dominado as pesquisas, pois mais de $90 \%$ dos estudos com nanopartículas para uso em remediação tem o nFeZ como material de análise. ${ }^{19}$ Evidencia-se a escolha pelo $\mathrm{nFeZ}$ devido sua baixa toxicidade e baixo custo de produção quando comparado a outras nanopartículas. Sua utilização tem se mostrado eficiente na desnitrificação microbiana e remoção de nitrato de águas subterrâneas, ${ }^{20}$ consistindo em um elemento muito reativo, caracterizado como agente redutor em grande número de poluentes. ${ }^{15}$ Ademais, apresenta potencial de aplicação combinada com processos de fitorremediação em solos contaminados com organoclorados. ${ }^{21}$

Considera-se também o uso do $\mathrm{nFeZ}$ no pré-tratamento para um subsequente processo de lavagem do solo afetado por elementos potencialmente tóxicos. A aplicação desse nanomaterial pode ser usado para imobilizar, sorver e capturar esses compostos, melhorando a lavagem do solo por adsorção de partículas, causando assim a formação de agregados maiores e mais pesados que são mais fáceis de separar devido a alta suscetibilidade magnética. ${ }^{22}$

A intensa discussão acerca da aplicação do nFeZ como tecnologia de remediação também levanta questões acerca dos riscos ambientais potenciais que a técnica pode trazer e que não são totalmente conhecidos. Por isso, a preocupação da pesquisa em analisar não apenas os resultados, mas também aspectos controversos relacionados, o que amplia o potencial dos estudos e a construção de um panorama científico sobre o tema. ${ }^{23}$

\section{REATIVIDADE DO nFeZ}

$\mathrm{O}$ nFeZ gera uma reação química de redução. No meio aquoso esta nanopartícula é suscetível à corrosão, sendo transformada rapidamente para $\mathrm{Fe}^{2+}$ e mais lentamente para $\mathrm{Fe}^{3+}$. Em águas com presença de oxigênio dissolvido (OD) o FeO, utiliza este elemento como oxidante (Equação 1). Seguindo a reação, ele pode transformar-se em $\mathrm{Fe}^{3+}$ (Equação 2), precipitando como um composto menos solúvel, o hidróxido de ferro. As reações também podem ocorrer em ambientes anaeróbios. Neste caso, o oxigênio da água é utilizado como elemento oxidante, produzindo hidrogênio na forma molecular (Equação 3). ${ }^{24}$

$$
\begin{gathered}
2 \mathrm{Fe}_{(\mathrm{s})}+\mathrm{O}_{2(\text { aq) }}+4 \mathrm{H}^{+}{ }_{\text {(aq) }} \rightarrow 2 \mathrm{Fe}^{2+}{ }_{(\mathrm{aq})}+2 \mathrm{H}_{2} \mathrm{O}_{(\mathrm{l})} \\
4 \mathrm{Fe}^{2+}{ }_{(\mathrm{aq})}+4 \mathrm{H}^{+}{ }_{(\mathrm{aq})}+\mathrm{O}_{2(\text { aq })} \rightarrow 4 \mathrm{Fe}^{3+}{ }_{(\mathrm{aq})}+2 \mathrm{H}_{2} \mathrm{O}_{(\mathrm{l})} \\
\mathrm{FeO}_{(\mathrm{s})}+2 \mathrm{H}_{2} \mathrm{O}_{(\mathrm{l})} \rightarrow \mathrm{Fe}^{2+}{ }_{(\mathrm{aq})}+\mathrm{H}_{2(\mathrm{~g})}+2 \mathrm{OH}^{-}
\end{gathered}
$$

A reação do ferro metálico $\mathrm{FeO}$ para o $\mathrm{Fe}^{2+}$ dissolvido tem um potencial padrão $\mathrm{E}^{0}$ de $-440 \mathrm{mV}$, indicando que o $\mathrm{FeO}$ tem grande capacidade de redução de contaminantes. Os processos mais comuns de redução de contaminantes orgânicos pelo $\mathrm{FeO}$ são hidrogenólise e dehalogenação. ${ }^{25}$

A hidrogenólise de compostos clorados, como o tricloroeteno (TCE), por exemplo, envolve a substituição de um átomo de cloro pelo átomo de hidrogênio, requerendo tanto um doador de elétron como um doador de próton (hidrogênio). A equação geral de redução de compostos clorados está apresentada na Equação 4. 26,27

$$
\mathrm{ClCH}=\mathrm{CCl}_{2}+2 \mathrm{e}^{-}+\mathrm{H}^{+} \rightarrow \mathrm{ClHC}=\mathrm{CHCl}+\mathrm{Cl}^{-}
$$

A dehalogenação envolve um processo redutivo de átomos de cloro sem a adição do hidrogênio, com a formação do uma nova ligação $C-C$, podendo ser ligado com um carbono vizinho (reação $\beta$ ) ou com o mesmo carbono (reação $\alpha$ ). Estas reações de dehalogenação estão apresentadas nas Equações 5 e $6 .{ }^{26}$

$$
\begin{gathered}
\text { Reação } \beta: \mathrm{ClHC}=\mathrm{CCl}_{2}+2 \mathrm{e}^{-} \rightarrow \mathrm{HC} \equiv \mathrm{CCl}+2 \mathrm{Cl}^{-} \\
\text {Reação } \alpha: \mathrm{Cl}_{2} \mathrm{C}=\mathrm{CH}_{2}+2 \mathrm{e}^{-} \rightarrow \mathrm{H}_{2} \mathrm{C}=\mathrm{C}:+2 \mathrm{Cl}^{-}
\end{gathered}
$$

Tem sido relatado que para a área de remediação de solos a cinética de reação de dehalogenação é favorável em relação à hidrogenólise pois, com esta última, geralmente existe a formação de compostos mais nocivos que o material de origem. Por exemplo, o tricloroeteno (TCE) quando reduzido por hidrogenólise forma o cisdicloroeteno (cDCE), cloroeteno, compostos bastantes nocivos; enquanto quando for reduzido por reação $\beta$ de dehalogenação forma o acetileno de cloro e acetileno, compostos muito instáveis que são rapidamente degradados. Estudos com $\mathrm{nFeZ}$ tem relatado a predominância da reação $\beta$ de dehalogenação, com a não formação do $\mathrm{CV}$, tornando este tipo de remediação vantajosa em relação a processos biológicos, onde a hidrogenólise é a reação predominante. ${ }^{28}$

Desde o processo da síntese para obtenção do $\mathrm{FeO}$, o mesmo reage com o oxigênio e forma uma fina camada de óxido de ferro em volta da partícula. Portanto, toda partícula de nFeZ é formada por um núcleo de $\mathrm{FeO}$ com uma crosta de óxido, com espessura de aproximadamente de 2 a $3 \mathrm{~nm} .{ }^{29}$ Quando aplicado na natureza, esta reação continua lentamente e a crosta vai aumentando de espessura, muitas vezes com a camada sendo formada por oxi-hidróxido de ferro $(\mathrm{FeOOH}) .{ }^{30}$ No entanto, esta fina camada de óxido ainda permite a 
transferência de elétrons do núcleo do metal, fazendo com que esta nanopartícula continue a apresentar capacidade de redução. Mas, pelo fato de ter uma crosta de óxido, a nanopartícula passa a ter também a capacidade de reagir com componentes inorgânicos, tais como aníons metálicos e metais.

A reação com contaminantes inorgânicos é controlada pelo potencial redox da espécie inorgânica. Se o contaminante tiver um potencial redox maior do que o $\mathrm{FeO}$ o componente é removido por redução e na sequência é precipitado ou coprecipitado. Se o potencial redox for muito menor do que do $\mathrm{FeO}$, a redução não será possível e o composto somente pode adsorvido nas nanopartículas. Existem situações em que ocorrerá tanto a redução quanto a coprecipitação.

A Tabela 1 apresenta o mecanismo de reação predominante do nFez que atuará nos contaminantes inorgânicos mais comuns. ${ }^{31}$

Tabela 1. Reação predominante do $\mathrm{nFeZ}$ nos contaminantes inorgânicos mais comuns

\begin{tabular}{lc}
\hline Reação predominante & Contaminante Inorgânico \\
\hline Redução & $\mathrm{Cr}, \mathrm{As}, \mathrm{Cu}, \mathrm{U}, \mathrm{Pb}, \mathrm{Ni}, \mathrm{Se}, \mathrm{Co}, \mathrm{Pd}, \mathrm{Pt}, \mathrm{Hg}, \mathrm{Ag}$ \\
Adsorção & $\mathrm{Cr}, \mathrm{As}, \mathrm{U}, \mathrm{Pb}, \mathrm{Ni}, \mathrm{Se}, \mathrm{Co}, \mathrm{Cd}, \mathrm{Zn}, \mathrm{Ba}$ \\
Oxidação/Reoxidação & $\mathrm{As}, \mathrm{U}, \mathrm{Se}, \mathrm{Pb}$ \\
Coprecipitação & $\mathrm{Cr}, \mathrm{As}, \mathrm{Ni}, \mathrm{Se}$ \\
Precipitação & $\mathrm{Cu}, \mathrm{Pb}, \mathrm{Cd}, \mathrm{Co}, \mathrm{Zn}$ \\
\hline
\end{tabular}

A adição de um segundo metal na superfície de nFeZ provoca um aumento significativo na reatividade da nanopartícula, pois este metal passa a ter a função de catalisador. Entre muitos metais de transição estudados para de halogenação catalítica, o paládio (Pd) é o mais comumente usado, devido às suas propriedades químicas e estruturais ideais para gerar espécies de hidrogênio e abrir caminho para quebra da cadeia de carbono-halogênio. No entanto, a adição deste segundo metal (espécie bimetálica) encarece o processo e torna a nanopartícula mais nociva ao meio ambiente e tem sido menos utilizado na aplicação prática de remediação. ${ }^{32}$

Para Yan et al..$^{19}$ a adição do segundo metal faz com que as reações de superfície da nanopartícula ocorram mais rapidamente, acelerando o processo de oxidação do $\mathrm{nFeZ}$ e, portanto, deixando a nanopartícula inativa em um período mais curto de tempo. Isto tem uma grande influência no transporte do contaminante em meio poroso, pois a efetividade do $\mathrm{nFeZ}$ será reduzida em relação à distância, exigindo mais pontos de aplicação no campo.

Todas as reações que ocorrem com as nanopartículas são fortemente influenciadas por um número grande de fatores, que dependem das características da própria partícula e, principalmente, das condições do local. Em outras palavras, as reações químicas são função das características do local de estudo, não podendo resultados de estudos obtidos em laboratório ser diretamente transferidos para a escala de campo. A realização de estudos em escala piloto ainda é necessária para se observar o real comportamento reativo da nanopartícula com o contaminante in situ. Para quem desejar se aprofundar no tema sobre reatividade do $\mathrm{nFeZ}$ recomenda-se a leitura do artigo publicado por Yan et al. ${ }^{19}$

Shi et $a l .{ }^{33}$ destacam em seu estudo que apesar da utilização de $\mathrm{nFeZ}$ para remediação de águas subterrâneas ser normalmente monitorada por medições comuns, tais como $\mathrm{pH}$, conteúdo de ferro total, e de oxidação e redução potencial, torna-se relevante considerar que essas medições podem ser enganosas por causa das complexas interações entre os materiais alvo (por exemplo, suspensões altamente reativas e variavelmente agregados nas nanopartículas) e materiais de aquíferos (sedimentos e águas subterrâneas), e várias complicações relacionadas aos métodos de amostragem e detecção. Por isso, os autores avaliam positivamente a utilização de medições mais específicas baseadas em análises químicas e eletroquímicas, aliado ao uso de sondas redox e métodos geofísicos.

Isso também é destacado por Chekli et al ${ }^{11}$ tendo em vista que em muitos estudos a mobilidade e reatividade do $\mathrm{nFeZ}$ em ambientes subsuperficiais pode ser afetada, pois dependem principalmente de propriedades tais como tamanho de partícula, composição química da superfície e composição em massa. Desse modo, a remediação eficaz deve aliar a avaliação precisa das variáveis envolvidas, compreendendo as implicações dessas propriedades antes de implantar esse nanomaterial em áreas contaminadas.

Há que se considerar que diferentes fatores interferem na reação e ação do nFeZ nos processos de remediação.

Xue et al. ${ }^{34}$ ressaltam que embora o $\mathrm{nFeZ}$ apresente benefícios potenciais e consideráveis nos processos de remediação, há uma necessidade distinta de identificar possíveis efeitos e riscos após a exposição ambiental desse nanomaterial, considerando diferenças nas metodologias e mecanismos de estabilização, fatores que afetam sua reatividade e que podem incluir área de superfície, $\mathrm{pH}$ do solo, matéria orgânica do solo, bactérias e fungos do solo e tipos de solo.

$\mathrm{O}$ pH desempenha um papel importante na reatividade, longevidade e seletividade do $\mathrm{nFeZ}$ para o contaminante alvo. A camada passiva da superfície do nanoferro em $\mathrm{pH}$ alto dificulta a transferência de elétrons, especialmente nos casos em que o poluentes é orgânico. ${ }^{35}$

Com relação ao solo, há diferenças nas formas como o nFeZ atua, a partir de características específicas do solo que afetam a adsorção e transformação nanomaterial. Agentes de dispersão e colóides naturais do solo afetam o transporte do $\mathrm{nFeZ}$ em meios porosos. ${ }^{36}$

A matéria orgânica também é um fator-chave que controla o destino e a mobilidade do $\mathrm{nFeZ}$ no solo por interações nos processos de adsorção, solubilidade e umidade. A matéria orgância é um mediador de elétrons, que facilita os elétrons transferir do $\mathrm{nFeZ}$ para contaminantes orgânicos e promover redução rápida de compostos orgânicos em solos. ${ }^{35}$

\section{O USO DE ESTABILIZANTES}

O nFeZ apresenta alta força de van der Waals, devido a sua alta Constante de Haymark e, também, apresenta propriedades magnéticas que causam a aglomeração das partículas em grumos, reduzindo significativamente a superfície específica. Além disso, as partículas são mais densas que a água, o que naturalmente já faz com que elas sedimentem no meio fluído. ${ }^{37}$ Estas propriedades tornam as nanopartículas difíceis de se deslocarem a grandes distâncias no subsolo, exigindo a construção de um número maior de pontos de injeção, encarecendo a técnica. Para Yan et al. ${ }^{19}$ a dificuldade de preparar suspensões de nFeZ estáveis e dispersas tem ainda sido um obstáculo para aplicações na prática.

A principal estratégia que tem sido adotada para aumentar a estabilidade de $\mathrm{nFeZ}$ é realizar a modificação das características das nanopartículas, reduzindo a sua reatividade e aumentando sua dispersão. A forma mais comum que tem sido utilizada é através da adição de produtos que "encobrem" as nanopartículas, técnica denominada coating. ${ }^{38} \mathrm{O}$ estabilizante pode ser adicionado no momento da síntese da partícula de $\mathrm{nFeZ}$, chamado de método pré-sintese, ${ }^{39,40}$ ou após, no momento da diluição, chamado de método de pós-síntese. ${ }^{41-43} \mathrm{O}$ método pós-síntese tem se mostrado eficiente para redução da reatividade das nanopartículas, ${ }^{44}$ enquanto o método pré-síntese tem dispersado as partículas, aumentando a área específica e, por consequência, a sua reatividade nanopartículas. ${ }^{39}$

Para o uso como estabilizante tem se preferido a utilização de compostos que tenham carga negativa, pois assim a reatividade com as partículas do solo (geralmente carregadas negativamente) é reduzida 
e, também, a aglomeração das partículas diminui devido à repulsão entre elas, garantindo a dispersão. ${ }^{45} \mathrm{O}$ estabilizante também deve ter características de fácil degradabilidade com baixa (ou nenhuma) toxicidade. ${ }^{46}$ Ou seja, o material estabilizante deve ser ambientalmente adequado para evitar que seu uso não seja mais um problema do que uma solução.

Os polímeros sintéticos foram os primeiros a serem utilizados como estabilizantes e demostraram bons resultados na redução da reatividade do $\mathrm{nFeZ}{ }^{41}$ Geralmente são adicionados em concentrações muito baixas, na ordem de alguns miligramas por litro. Existem vários tipos de polímeros que estão descritos na literatura, entre eles destacam-se: ácido poliacrílico, ${ }^{38,43}$ poliestireno sulfonato e poliaspartato, ${ }^{44}$ e a mistura de diferentes polieletrólitos. ${ }^{47}$ Camessele et al., ${ }^{43}$ além de estudarem alguns polímeros (ácido poliacrílico e ácido aspártico), avaliaram também a utilização de lactato de alumínio, lactato de sódio e lactato etílico, bem como diferentes composições de ciclodextrina. Observaram que estes compostos são adequados para utilização como dispersantes, sendo o lactato de alumínio o que apresentou o melhor resultado. Para Yan et al. ${ }^{19} \mathrm{o}$ ácido poliacrílico e o poliestireno sulfonato são os polímeros mais atrativos para aplicação devido ao seu elevado peso molecular e alta densidade de grupos funcionais presentes na molécula.

Tosco et al.$^{48}$ descrevem que o uso de polímeros naturais (biopolímeros) tem despertado grande interesse do meio técnico, devido à grande disponibilidade, o baixo valor comercial e a pouca nocividade ao meio ambiente. Já foram utilizados os seguintes biopolímeros: goma guar, ${ }^{42,49}$ goma xanthan, ${ }^{50}$ alginato de cálcio,${ }^{51}$ amido,${ }^{52}$ carboximetil-celulose $\mathrm{e}^{39,53-55}$ e extrato de resíduo de seriguela. ${ }^{56}$

Uma forma alternativa proposta para estabilizar o $\mathrm{nFeZ}$ para uso com contaminantes mais pesados que água (DNAPL) foi adicioná-lo em emulsões de água-óleo. Os óleos utilizados são misturados com surfactante e são biodegradáveis, tais como óleo de soja e óleo de milho. A técnica consiste em encapsular partículas de $\mathrm{nFeZ}$ em óleo não permitindo, portanto, a sua interação com água subterrânea. Isto permite que o $\mathrm{nFeZ}$ encapsulado em uma bolha de óleo chegue a profundidades maiores sem reagir. Estudo realizado em campo, onde foi aplicada esta técnica para reagir com DNAPL, mostrou bons resultados..$^{57-59}$

Outra forma de aumentar a estabilidade das nanopartículas é aderindo as mesmas em um substrato sólido, tornando-os materiais compósitos. Os principais substratos estudados foram: sílica, ${ }^{60}$ carbonos, ${ }^{61,62}$ resinas, ${ }^{63,64}$ bentonita ${ }^{65}$ caulinita ${ }^{66}$ e zeolite.${ }^{67}$ Estes estudos foram todos realizados em escala de bancada, sendo necessário estudo em campo para avaliar a eficiência. Para Yan et al. ${ }^{19}$ pesquisas são necessárias para produzir estes compostos em larga escala e com preços competitivos.

Su et al.$^{68}$ propôs a estabilização do nanoferro de valência zero a partir do processo de biomassa carbonizada (biochar) com base em ensaios de sedimentação e de experiências de coluna. Os resultados mostraram que ocorre menor agregação, mantendo a realitivada das nanopartículas de $\mathrm{nFeZ}$, melhor estabilidade e mobilidade, bem como redução da lixiviação do ferro no solo. Pei et al. ${ }^{69}$ comprovaram que um material composto por $n F e Z$ e resíduo de vinagre pode ser utilizado nos processos de remediação de solos contaminados por cromo hexavalente $(\mathrm{Cr}(\mathrm{VI}))$.

\section{MECANISMOS DE TRANSPORTE DO nFeZ}

Muitos estudos estão sendo realizados a fim de entender como as nanopartículas reagem com o contaminante e migram em um meio poroso. Estes estudos estão sendo realizados em laboratório através de ensaios em coluna, os quais são importantes para avaliar como é a interação do contaminante com as nanopartículas e, também, para se ter ideia da sua mobilidade. No entanto, existem poucos dados obtidos em pesquisas que avaliaram de forma sistemática o transporte de nanopartículas em escala de campo. ${ }^{48}$

Ensaios de coluna realizado com $\mathrm{nFeZ}$ sem ser estabilizado mostrou pequena mobilidade no meio poroso (alguns centímetros), não sendo, portanto, efetiva sua aplicação no solo sem o uso de um estabilizante. ${ }^{70-73} \mathrm{~A}$ pouca mobilidade destas partículas foi atribuída à filtração mecânica dos agregados de nanopartículas formados devido às forças de atração. ${ }^{74}$ Para Reddy ${ }^{37}$ o transporte de nanopartículas pode ser impedido, além da componente mecânica, pelas interações hidrofílicas e hidrofóbicas, pela heterogeneidade do meio e pelas reações químicas das nanopartículas com as partículas sólidas dos solos.

O uso de compostos estabilizantes em partículas de nFeZ mostrou-se efetivo em vários estudos de colunas. Partículas que sem tratamento migravam alguns centímetros no meio poroso, após o tratamento conseguiram ser transportadas atravessando toda a coluna. ${ }^{42,53,73-76}$ Resultados interessantes mostram que a matéria orgânica naturalmente encontrada em alguns solos apresenta ação similar aos estabilizantes adicionados a $\mathrm{nFeZ}$, aumentando a mobilidade dessas nanopartículas. ${ }^{72}$

O'Carrol et al..$^{31}$ reconhece que grandes avanços foram obtidos para entender a migração de contaminantes através de ensaios de coluna, mas destaca que muitos destes estudos foram realizados em condições que não representam a realidade de campo. Principalmente, questões relacionadas com a velocidade de fluxo, onde geralmente foram adotados gradientes hidráulicos muitos superiores aos utilizados em injeções no campo. Outro fato relevante que os autores destacam está relacionado com a questão de concentração de nanopartículas, que é representada na modelagem através da viscosidade do líquido injetado; em muitos estudos foram adotados valores muitos abaixo da real necessidade para reagir com o contaminante. Reddy ${ }^{37}$ já havia levantado esta questão e também apontava para a pouca representatividade dos solos reais nos ensaios de coluna, já que o meio avaliado geralmente são areias limpas ou partículas de vidro, com pouca ou nenhuma reatividade com o líquido que está percolando. Além disso, a utilização de água destilada (ou deionizada) como líquido condutor das nanopartículas não reproduz as reações que ocorrerão com a água subterrânea in situ.

A migração de nanopartículas no meio poroso vai depender, portanto, de variáveis como velocidade de injeção, distribuição granulométrica do meio, viscosidade da solução injetada (concentração) e reatividade do meio (água subterrânea e partículas de solo). Estudos iniciais em coluna que estão sendo realizados pelos autores deste artigo com solo residual de basalto do Brasil (Latossolo), estão indicando que a concentração máxima de $\mathrm{nFeZ}$ não estabilizado que pode ser utilizado para não ocorrer a colmatação dos poros é de aproximadamente $4 \mathrm{~g} \mathrm{~L}^{-1}$. Já quando utiliza-se $\mathrm{nFeZ}$ estabilizado, a concentração máxima aumenta para aproximadamente $10 \mathrm{~g} \mathrm{~L}^{-1}$. $\mathrm{O}$ estudo está indicando que apesar da alta porosidade dos solos residuais, a concentração de nFeZ que pode ser utilizada não é muito elevada.

Reddy et al. ${ }^{77}$ avaliaram o uso da técnica de eletrocinética para melhorar a migração de nanopartículas em meios porosos de baixa permeabilidade (argilas sedimentares). Foram utilizados ensaios em laboratório usando o caulin como meio poroso percolado com nFeZ na forma pura e $\mathrm{nFeZ}$ estabilizado com lactato de alumínio. Verificaram que a técnica de eletrocinética melhorou a migração das partículas em meio poroso de baixa permeabilidade, podendo viabilizar o uso da nanoremediação também nestes tipos de materiais.

Os mecanismos envolvidos para retenção de coloides pelo meio poroso são classificados como físicos e físico-químicos. ${ }^{48} \mathrm{O}$ processo de filtração ou retenção das partículas é um processo predominante físico. A retenção de nanopartículas individuais é pouco provável, uma 
vez que o tamanho das nanopartículas é muito inferior ao tamanho dos poros que permitem a passagem do líquido. ${ }^{78}$

As interações físico-químicas entre as nanopartículas e o meio poroso resultam em deposições dinâmicas que podem ser reversíveis. Este processo pode variar com o decorrer do tempo, sendo que no início da injeção poucas nanopartículas são depositadas sobre a superfície das partículas do solo, possuindo pouca interferência. No entanto, com o passar do tempo, um número maior de partículas vai se aderindo ao solo, reduzindo os espaços entre eles, passando a exercer a função de filtros, ou seja, retendo nanopartículas que vem chegando ao local, além de começar a influenciar o equilíbrio eletroquímico. No final do processo esta interação acaba por colmatar totalmente os espaços reduzindo e, algumas vezes, até evitando o fluxo de líquido pelo local. Portanto, o fluxo e o transporte de nanopartículas são problemas acoplados. ${ }^{78,79}$ Esta é a principal diferença no mecanismo de transporte de nanopartículas em meios porosos em relação ao transporte de contaminantes. No caso de transporte de nanopartículas o processo advectivo acaba sendo influenciado ao longo do tempo, fato que não ocorre no transporte de contaminantes.

Para modelar o transporte de nanopartículas têm sido utilizados modelos baseados na teoria de filtração de coloides proposta por Yao et al.$^{80}$ Esta teoria é incorporada na equação de advecção e dispersão de transporte de contaminantes, incluindo um terceiro termo que procura descrever a filtração físico-química que ocorre no meio poroso (Equação 7). ${ }^{73}$

$$
\mathrm{R} \frac{\partial \mathrm{C}}{\partial \mathrm{t}}=\mathrm{Dh} \frac{\partial^{2} \mathrm{C}}{\partial^{2} \mathrm{x}}-\mathrm{Vp} \frac{\partial \mathrm{C}}{\partial \mathrm{x}}-\lambda \mathrm{C}
$$

Onde C é a concentração da nanopartícula, $\mathrm{R}$ é coeficiente de retardamento, t é o tempo, Dh é o coeficiente de dispersão, Vp é a velocidade do fluído, $\mathrm{x}$ é o comprimento de fluxo, e $\lambda$ é constante de primeira ordem que leva em consideração a filtração, ou seja, a razão de quanto as nanopartículas são aderidas ou depositadas no sistema coletor ao passarem por ele. $\mathrm{O}$ valor de $\lambda$ é calculado considerando o efeito acumulativo da difusão (considerando a suspensão com características de líquido Newtoniano), interceptação e a ação da gravidade sobre as partículas que passam o filtro.

O modelo de filtração foi utilizado por diversos pesquisadores para reprodução de resultados de ensaios de coluna com sucesso. ${ }^{44,50,81}$ Alguns trabalhos usaram estes resultados para estimar a distância que as nanopartículas migrariam em situações in situ. ${ }^{82-84}$ No entanto, as concentrações de $\mathrm{nFeZ}$ utilizadas em aplicações de campo são superiores às aplicadas nos estudos de coluna, fazendo com que a aplicação direta dos resultados deva ser analisada com precaução. ${ }^{48}$ Alterações no modelo de filtração tem sido propostas, levando em consideração questões relacionadas à interação magnética entre as nanopartículas e as propriedades não newtonianas da suspensão. ${ }^{31,85}$

Alguns modelos diferentes do de filtração foram avaliados para serem aplicados no transporte de nanopartículas. Quando não ocorre a colmatação dos poros, ou seja, quando a concentração de nanopartículas é pequena, foi possível a utilização dos modelos HYDRUS ${ }^{86}$ (2005), STANMOD ${ }^{87}$ e MNM1D. ${ }^{48}$ O modelo SEAWAT, que leva em consideração a variação da densidade das partículas, foi utilizado para avaliar transporte em 2D de nanopartículas em areias. ${ }^{53}$ Tosco e Sethi ${ }^{78}$ propuseram um novo modelo, chamado E-MNM1D, que leva em consideração as propriedades visco-plásticas do fluído de transporte e a progressiva colmatação do meio poroso.

Existe um consenso entre os pesquisadores que trabalham para entender o transporte de nanopartículas que existe a necessidade de ampliar os trabalhos para modelagem de trabalhos em escala real. Ainda é necessário incluir nos modelos várias questões não abordadas até o momento, tais como: as reações entre as nanopartículas, reatividade com o meio (solo e água subterrânea), reatividade com os contaminantes e a previsão de concentração residual no meio ambiente no final do processo. ${ }^{31,37,85}$

\section{CONTAMINANTES}

Devido a alta reatividade, tamanho reduzido e grande especificidade do $\mathrm{nFeZ}$ esta nanopartícula pode transformar vários contaminantes, como metais pesados, compostos orgânicos clorados e compostos inorgânicos. ${ }^{88}$

A remediação de solos exige que o agente descontaminante seja adicionado diretamente no meio, sendo sua remoção difícil uma vez que ocorre contato da biota com o material além da possibilidade deste ser arrastado até as águas subterrâneas. Neste caso, o nFeZ surge como material adequado a ser empregado neste meio, principalmente por diminuir a toxicidade de solos contaminados com cromo, arsênio, bem como na redução de compostos organoclorados. ${ }^{89}$

Desde o trabalho publicado por Wang e Zhang, ${ }^{17}$ que utilizaram nanopartículas bimetálicas para descontaminar água com solventes clorados, muitos trabalhos foram realizados com uma grande variedade de contaminantes orgânicos e inorgânicos. Um estudo realizado por Yan et al. ${ }^{19}$ baseado em 445 publicações, mostrou que os principais tipos de contaminantes que foram tratados com $\mathrm{nFeZ}$ foram os seguintes: halogenados alifáticos $(26,9 \%)$, halogenados aromáticos (17,8\%), outros componentes orgânicos $(17,8 \%)$, metais $(25,8 \%)$ e não metais inorgânicos $(11,7 \%)$. Portanto, o tratamento de contaminantes orgânicos predominou nas pesquisas, correspondendo a $62,5 \%$ do total. Os contaminantes inorgânicos correspondem a $37,5 \%$, com destaque para os contaminantes metálicos, que foram os mais estudados nesta categoria. Brandl et al..$^{90}$ demonstram uma experiência piloto em pequena escala sobre águas residuais e solos contaminados onde o uso de nanopartículas mostrou-se eficaz na redução da teratogenicidade do bisfenol A, o triclosan e o estradiol, sem gerar subprodutos tóxicos.

Danish et al. ${ }^{91}$ ao avaliarem o desempenho do $\mathrm{nFeZ}$ catalisado por percarbonato de sódio em um sistema heterogêneo sobre a degradação de organoclorados do tipo tricoroetano e tricloroetileno, verificaram que o mesmo tem grande potencial para degradar tais contaminantes mostrando-se como importante técnica de remediação da água subterrânea contaminada.

\section{TOXICIDADE E QUESTÕES CONTROVERSAS DO USO DE $n F e Z$}

Apesar da pesquisa envolvendo as nanopartículas estar em expansão, o conceito de injetar deliberadamente essas substâncias no subsolo, embora seja para destruição e transformação de contaminantes comprovadamente nocivos à saúde humana, tem gerado questionamentos e preocupações acerca de seu potencial tóxico. ${ }^{46}$

No caso do nFeZ, as mesmas características que o tornam um excelente material para aplicação em descontaminação do solo e água subterrânea, tais como pequeno tamanho e alta capacidade de redução e oxidação, também fazem dele um potencial contaminante do meio ambiente..$^{92}$

Apesar da nanoremediação ter potencial para limpar grandes locais contaminados, reduzindo o tempo e a concentração do contaminante, torna-se importante implementar as ações de pesquisa e análise em cenários da vida real para se evitar efeitos deletérios. ${ }^{13}$ A compreensão do potencial de remediação ambiental do nFeZ não pode se limitar a experimentos laboratoriais, mas incluir estudos piloto e de campo. ${ }^{11}$

Análise da toxicidade do $\mathrm{nFeZ}$ em micro-organismos foram realizados em escala de bancada, mostrando que a nanopartícula tem um alto potencial biocida quando usado sem estabilizante. No 
entanto, após o nFeZ tornar-se oxidado, sua influência sobre os micro-organismos se inverte, tornando-o um excelente estimulante da atividade microbiológica pela biodisponibilidade do ferro oxidado. ${ }^{93}$

Para Tosco et al., ${ }^{48}$ este comportamento é favorável ao uso em remediação, pois no início o ferro é utilizado como redutor do contaminante e, após a sua oxidação, ele auxiliará na descontaminação que por acaso persistir, através da bioestimulação. Também foi observado que o uso de $\mathrm{nFeZ}$ com estabilizantes orgânicos auxilia no processo de bioremediação, já que o estabilizante atua como um bioestimulante. Por outro lado, Li e Zhang ${ }^{94}$ encontraram que a concentração do $\mathrm{nFeZ}$ sem estabilizante para ter efeito biocida era de $5 \mathrm{mg} \mathrm{L}^{-1}$ de nFeZ, enquanto com $\mathrm{nFeZ}$ estabilizado a concentração aumentava para $500 \mathrm{mg} \mathrm{L}^{-1}$, ou seja, 100 vezes maior. Apesar desta grande diferença, a nanopartícula estabilizada ainda apresentou efeito biocida.

Segundo Crane e Scott,${ }^{95}$ a injeção de $\mathrm{nFeZ}$ na água subterrânea in situ vai apresentar uma toxicidade menor do que tem apresentado em escala in vitro, devido às rápidas reações de oxidação que ocorrem. Para os autores, as chances de ocorrer efeitos tóxicos a seres humanos ou outros mamíferos, proveniente de água subterrânea, é pouco provável uma vez que as reações de oxidação do ferro irão ocorrer muito antes de qualquer possibilidade de contato. A contaminação de $\mathrm{nFeZ}$ em humanos pode ocorrer mais provavelmente pelo manuseio (contato com pele) e inalação, antes da injeção no subsolo, podendo ser evitável através do uso de equipamentos de segurança.

A hipótese de que a toxicidade do nFeZ em aplicações ambientais ainda não é conhecida. ${ }^{96} \mathrm{O}$ fato do ferro metálico contribuir para melhorar o processo de transferência de elétrons e para aumentar o sobrepotencial de reações interferentes paralelas (competição pelos elétrons, desprendimento de hidrogênio e diminuição da eficiência da reação de degradação dos poluentes alvos), exige a necessidade de ampliar o estudo dos efeitos tóxicos que podem ser resultados dessas ações. ${ }^{97}$

Estudo de Anza et al. ${ }^{98}$ identificou que o nFeZ impacta na atividade microbiana do solo. Os autores pesquisaram a ação do nanomaterial sobre um solo contaminado artificialmente com lindano e zinco. A aplicação de nFeZ reduziu as concentrações desses elementos, mas também reduziu a biomassa e a atividade microbiana do solo.

A citotoxicidade do $\mathrm{nFeZ}$ está relacionada a ruptura da membrana celular e estresse oxidativo gerado através de espécies de $\mathrm{Fe}^{2+}$ e oxigênio reativo pelo nanomaterial, capazes de alterar substancialmente a composição taxonômica e funcional das comunidades microbianas presentes no solo. ${ }^{88}$

Com relação aos efeitos de toxicidade, Cecchin et al.${ }^{99}$ avaliaram a produção de $\mathrm{CO}_{2}$ em microrganismos nativos de um solo nacional (Passo Fundo) a partir da aplicação de diferentes concentrações de $\mathrm{nFeZ}\left(0,4,15\right.$ e $\left.50 \mathrm{~g} \mathrm{~kg}^{-1}\right)$. Os autores utilizaram como fonte de carbono a glicose $\left(\mathrm{g} \mathrm{kg}^{-1}\right)$, sendo que os resultados mostraram que na concentração de $50 \mathrm{~g} \mathrm{~kg}^{-1}$ não ocorreu liberção de dióxido de carbono, demonstrando má adaptação dos microrganismos nessas condições. As concentrações de 4 e $15 \mathrm{~g} \mathrm{~kg}^{-1}$ de nFeZ mostraram um aumento na produção de $\mathrm{CO}_{2}$, indicaram adaptação dos microrganismos, o que denota que aplicações mais altas podem causar efeitos tóxicos e/ou inibitórios à atividade microbiana, restringindo uma possível combinação de nano e biorremediação.

Apesar de ser promissor o uso de nanomateriais utilizados para remediação de solo e água subterrânea, incluindo nanocompostos multifuncionais para sequestro e degradação de contaminantes sinérgicos, adsorventes seletivos e catalisadores, bem como reagentes de liberação lenta, verificam-se que ainda há dúvidas quanto aos limites de misturas e transporte de nanomateriais, o que pode restringir as aplicações in situ desses materiais. ${ }^{36}$

A agregação e acumulação de nFeZ tem sido relatada como as principais desvantagens para sua aplicação, sendo que sua modificação pode melhorar e melhorar sua estrutura, mobilidade e aplicação no ambiente. ${ }^{100}$

A partir de uma perspectiva funcional, a introdução do $\mathrm{nFeZ}$ no meio ambiente provavelmente perturbará o equilíbrio entre grupos microbianos funcionais que realizam importantes processos biogeoquímicos no solo. Além disso, é necessário ampliar a discussão acerca de como as aplicações in situ do $\mathrm{nFeZ}$ poderá afetar ecossistemas para onde não foram direcionadas. ${ }^{88}$

Outra questão é que apesar do nFeZ ter elevada área superficial e reatividade, aumentando assim a eficiência da remoção de poluentes alvo, a corrosão de $\mathrm{FeO}$ é produzida a partir da atividade anaeróbica do $\mathrm{nFeZ}$ precipitado na superfície da partícula, inibindo a atividade do nanomaterial e pode limitar sua aplicação nos processos de remediação ambiental. ${ }^{101}$

Assim, memo com os resultados obtidos acerca da efetividade da ação do $\mathrm{nFeZ}$ na descontaminação de produtos altamente nocivos, comprovando sua viabilidade de uso, os riscos toxicológicos ainda devem ser alvo de estudo, sendo esta uma área de estudo e pesquisa que necessita ser avaliada para que a tecnologia torne-se segura. ${ }^{48}$

\section{VIABILIDADE DO USO DA NANOTECNOLOGIA}

O primeiro uso de nanopartículas em aplicação in situ aconteceu no início da década passada, ${ }^{102}$ e desde então tem-se ampliado os estudos para o desenvolvimento da técnica. Atualmente se sabe que a aplicação de nanopartículas não estabilizadas tem baixa mobilidade e o uso de estabilizantes melhora a mobilidade também em aplicações in situ. . $^{54103-106}$

A viabilidade do uso da nanotecnologia é foco de diversos estudos de revisão realizados nos EUA ${ }^{5}$ e Europa. ${ }^{107}$ Detalhes dos locais também podem ser encontrados nas páginas da internet que abordam os projetos sobre nanotecnologias emergentes. ${ }^{108,109}$ Quanto aos contaminantes que podem ser alvo da tecnologia destaque para diferentes substâncias, incluindo organoclorados com destaque para PCE, TCE e PCB; bem como outros tais como Cr, Ni e Nitratos. A eficiência da técnica é verificada a partir de uma variação de $40 \%$ a $99 \%$, com predominância de valores acima de $90 \%$. A concentração de $\mathrm{nFeZ}$ utilizada variou de, no mínimo $1 \mathrm{~g} \mathrm{~L}^{-1}$ até o máximo de $30 \mathrm{~g} \mathrm{~L}^{-1}$, predominando valores até $10 \mathrm{~g} \mathrm{~L}^{-1} \cdot{ }^{19,110,111}$

Esses dados apontam para que o uso do $\mathrm{nFeZ}$ consolide-se como viável nos processos de remediação. Nos trabalhos in situ podem ser utilizados diferentes métodos para injeção das nanopartículas no meio, dependendo basicamente da permeabilidade do mesmo. Nos locais onde os materiais são permeáveis e existe pequeno volume de solo contaminado, a injeção pode ocorrer através da infiltração por poços escavados no local (piezômetros) ou hastes cravadas no terreno (direct push). Neste caso, a pressão de injeção é aquela da coluna de líquido no sistema. Para materiais que tem permeabilidade mais baixa e onde é necessário aumentar a distância de distribuição, deve-se utilizar a injeção sob pressão, que pode ou não gerar o fraturamento do meio, dependendo da pressão adotada. ${ }^{112,113}$ Para solos de baixa permeabilidade geralmente são adotadas pressões elevadas a fim de ocorrer o fraturamento do meio, facilitando a percolação.

Ainda não existem procedimentos padrão para elaboração de projeto a fim de definir o número de poços de injeção, a pressão que deve ser utilizada, a distância de cada poço, a concentração de nanopartículas e a distância e tempo de percolação da mistura. Todos estes elementos são específicos de cada local a ser tratado, não sendo possível utilizar dados de bibliografias para realização de projetos. O recomendado é a realização de ensaios de laboratório com solo e água do local a fim de obter parâmetros para tomada de decisões de projetos e, após, realizar estudos em escala piloto. Com os dados destes estudos em escalar menor é possível avaliar o real 
comportamento da técnica in situ e verificar se as variáveis adotadas no projeto foram corretas ou necessitam de alterações. . $^{19,95}$

O custo da aplicação da nanotecnologia na remediação de solos depende de diversos fatores, como custo da matéria prima, injeção e monitoramento. O custo do $\mathrm{nFeZ}$ tem variado devido ao aumento da produção e do número de fornecedores. Valores do ano 2004 relatavam custos de aproximadamente $\$ 200,00 / \mathrm{kg}$ de $\mathrm{nFeZ} .{ }^{95} \mathrm{Em} 2019$, o valor médio do nFeZ é de $€ 128 / \mathrm{kg}$. ${ }^{14}$

Os custos de injeção e monitoramento são específicos de cada local, dependendo da complexidade da geologia, da profundidade do contaminante, do tamanho da área tratada e do tempo necessário de monitoramento após a aplicação da técnica. Nas 58 aplicações in situ, poucos foram os trabalhos que relatam o valor médio por unidade de volume tratado. Os valores apresentados na literatura variaram de $\$ 130,00$ a $\$ 320,00 / \mathrm{m}^{3}$ de solo contaminado. ${ }^{110}$

Karn et al..$^{5}$ relatam que a aplicação da nanotecnologia em águas subterrâneas contaminadas, em substituição à técnica de bombear e tratar ( pump and treat) que normalmente é adotada pode reduzir os custos do tratamento de 80 a $90 \%$. Relatam, também, que somente nos EUA a economia pela substituição da técnica seria de aproximadamente 90 bilhões de dólares nos próximos 30 anos.

Dong et al. ${ }^{101}$ sugerem maiores estudos acerca da ação do $\mathrm{nFeZ}$ na remediação de águas contaminadas, considerando a necessidade de ampliar a pesquisa acerca das interações orgânicas e inorgâncias que coexistem no ambiente subterrâneo. Nessa perspectiva, cabe explorar o desempenho operacional do $\mathrm{nFeZ}$ a fatores como dosagem, $\mathrm{pH}$ e temperatura, além de que a coexistência de diferentes microorganismos geram uma infinidade de interações e afetam o efeito do $\mathrm{nFeZ}$ na remoção de contaminantes.

Pesquisas realizadas com solos nacionais já estão sendo desenvolvidas. O estudo de Cecchin et al. ${ }^{115}$ analisou a ação do $\mathrm{nFeZ} \mathrm{em}$ solo contaminado com biodiesel, verificando uma rápida degradação inicial do contaminante (entre $17,9 \%$ a $32,03 \%$ ), o que comprova a eficácia do nanomaterial na remediação, mas uma redução drástica do processo degradador após 192 horas.

No entanto, grande parte da pesquisa é função do local contaminado, ou seja, não é possível transferir diretamente resultados obtidos em solos sedimentares de regiões de clima temperado, locais onde grande parte dos estudos apresentados na literatura são desenvolvidos, para solos com características únicas como são solos brasileiros de regiões tropicais e subtropicais. Fica evidente que existe a necessidade do desenvolvimento de uma nanotecnologia nacional para aplicação em remediação de solos e águas subterrâneas contaminadas.

Quanto à síntese de nanopartículas, já existem empresas que estão produzindo tanto nanopartículas não estabilizadas como estabilizadas. O desafio no Brasil é produzir esses nanomateriais com valores competitivos aos produzidos no mercado internacional. Um tema de grande interesse é o relacionado aos reagentes polifenólicos ecológicos que, em tese, podem ser injetados diretamente no solo para reagir com o ferro dissolvido em águas subterrâneas ou com ferro presente nos solos residuais. Ainda, existe o desafio de obter os extratos de outras matérias-primas ainda não estudadas e que existem nas diferentes regiões do país.

O desenvolvimento de estabilizantes orgânicos para uso em pós-síntese de partícula a fim de melhorar a estabilidade das nanopartículas, a partir de produtos nacionais de baixo custo, é um desafio que deve ser buscado pelas pesquisas.

Em relação à reatividade, o transporte e a toxicologia nos solos residuais brasileiros, pesquisas estão sendo desenvolvidas. Contudo, é necessária a realização tanto de estudos básicos em laboratório, como estudos em campos experimentais, antes da aplicação da técnica em escala real. Nas aplicações in situ a realização de monitoramento sistemático e em longo prazo será necessário. Ademais, considera-se necessária muita pesquisa sobre ecotoxicidade de nanopartículas, uma vez que o solo é uma matriz ambiental muito complexa. ${ }^{98}$

Enfim, é possível concluir que a técnica de remediação utilizando nanopartículas é complexa e influenciada por diversas questões bio-físico-químicas do local contaminado. Embora vários resultados impressionantes já apontam para o sucesso da remediação de solos e água utilizando $\mathrm{nFeZ}$, pesquisas precisam ser ampliadas, especialmente a partir da combinação com a bio-remediação, tornando mais significativo e competitivo esse sistema. ${ }^{35}$

Portanto, a técnica tem se mostrado promissora para tratar diferentes contaminantes, principalmente considerando aspectos relacionados a custos. O Brasil precisa aproveitar os conhecimentos já adquiridos e adaptá-los para condições específicas de solo e clima brasileiro. Assim será possível viabilizar, totalmente, o uso da técnica em um futuro próximo.

\section{CONSIDERAÇÕES FINAIS}

A nanotecnologia tem se mostrado eficiente para descontaminação de uma gama de contaminantes que atingem o solo e a água, a um custo competitivo. Especialmente o $\mathrm{nFeZ}$ tem sido amplamente pesquisado como um dos principais nanomateriais que podem ter alto potencial para remediação.

Nesta revisão, buscou-se compreender como o nFeZ age e interage nos solos e águas contaminadas, possibilitando a redução de contaminantes. A literatura apresenta resultados promissores com relação ao seu uso. Contudo, ainda é necessário avançar nas pesquisas de campo e em larga escala, uma vez que a maioria dos estudos se concentra no âmbito laboratorial. Nesse sentido, é importante incluir a análise da toxicidade desse nanomaterial, bem como sua reatividade frente às diferentes variáveis que fazem parte da atividade de remediação, promovendo assim a técnica e sua aplicabilidade como tecnologia ambiental.

Considera-se necessário o estudo do comportamento do $\mathrm{nFeZ}$ nos solos nacionais como forma de adequar as metodologias de uso, bem como sua performance e ação ao longo do tempo. Além disso, a ampliação da pesquisa no Brasil poderá trazer novas possibilidades, minimização de custos, maior segurança e efetivação de ações para ampliar as atividades de remediação de solos e águas contaminadas, trazendo melhores perspectivas à questão ambiental.

\section{REFERÊNCIAS}

1. Higueras, P. L.; Sáez-Martínez, F. J.; Reyes-Bozo, L.; Environ. Sci. Pollut. Res. 2016, 23, 5997.

2. U.S. Environmental Protection Agency (USEPA); Contaminated land, 2020. (disponível em https://www.epa.gov/report-environment/ contaminated-land, acessada em Janeiro 2020).

3. Pérez, A. P.; Eugenio, N. R.; JRC Technical Reports, 2018. (disponível em https://publications.jrc.ec.europa.eu/repository/bitstream/ JRC107508/jrc107508_2018.1264_src_final_progress_in_the_ management_contaminated_sites_in_europe_eur_29124_en_onlinefinal_1.pdf, acessada em Janeiro 2020).

4. CETESB; Relatório de áreas contaminadas e reabilitadas no Estado de São Paulo, 2018. (disponível em https://cetesb.sp.gov.br/areascontaminadas/wp-content/uploads/sites/17/2019/04/Texto-explicativo_ dez-2018.pdf, acessada em Novembro 2019).

5. Karn, B.; Kuiken, T.; Otto, M.; Cien. Saude Colet. 2011, 16, 165.

6. Reddy, K. R.; Environmental Geotechnics 2013, 1, 136. (disponível em http://dx.doi.org/10.1680/envgeo.13.00088, acessada em Novembro 2019).

7. Ferreira, H. S.; Rangel, M. C.; Quim. Nova 2009, 32, 1860.

8. Quina, F. H.; Quim. Nova 2004, 27, 1028. 
9. Cundy, A. B.; Hopkinson, L.; Whitby, R. L. D.; Sci. Total Environ. 2008, $400,42$.

10. Araújo, R.; Castro, A. C. M.; Fiúza, A.; Mater. Today: Proc. 2015, 2, 315.

11. Chekli, L.; Bayatsarmadi, B.; Sekine, R.; Sarkar, B.; Shen, A. M.; Scheckel, K. G.; Skinner, W.; Naidu, R.; Shon, H. K.; Lombi, E.; Donner, E.; Anal. Chim. Acta 2016, 903, 13.

12. Faria, E. M.; Eleamen, G. R. A.; Silva, A. E.; Mendonça, E. A. M.; Oliveira, E. E.; Biofar, Rev. Biol. Farm. 2013, 9, 18.

13. Rajan, C. S. J.; Env. Sci. Dev. 2011, $2,1$.

14. Thomé, A.; Reddy, K. R.; Reginatto, C.; Cecchin, I.; Water, Air, Soil Pollut. 2015, 226, 121.

15. Cabello, G.; Gromboni, M. F.; Pereira, E. C.; Marken, F.; Electrochem. Commun. 2016, 62, 48.

16. Poursaberi, T.; Konoz, E.; Sarrafi, A. H. M.; Hassanisadi, M.; Hajifathli, F.; Chem. Sci. Trans. 2013, 1, 658

17. Wang, C. B.; Zhang, W. X.; Environ. Sci. Technol. 1997, 31, 2154.

18. Ponder, S. M.; Darab, J. G.; Mallouk, T. E.; Environ. Sci. Technol. 2000, 34, 2564.

19. Yan, W.; Lien, H.-L.; Koel, B. E.; Zhang, W.-X.; Environ. Sci.: Processes Impacts 2013, 15, 63.

20. Peng, L.; Liu, Y.; Gao, S. H.; Chen, X.; Xin, P.; Dai, X.; Ni, B.; Sci. Rep. 2015, 5,12331

21. Pillai, H. P. S.; Kottekottil, J.; J. Environ. Prot. 2016, 7, 734.

22. Boente, C.; Sierra, C.; Martínez-Blanco, D.; Menéndez-Aguado, J. M.; Gallego, J. R.; J. Hazard. Mater. 2018, 350, 55.

23. Vanzetto, G. V.; Thomé, A.; Environ. Pollut. 2019, 252, 74

24. Kharisov, B. I.; Dias, H. V. R.; Kharissova, O. V.; Jimenez-Perez, V. M.; Perez, B. O.; Flores, B. M.; RSC Adv. 2012, 2, 9325.

25. Arnold, W. A.; Roberts, A. L.; Environ. Sci. Technol. 2000, 34, 1794.

26. Matheson, L. J.; Tratnyek, P. G.; Environ. Sci. Technol. 1994, 28, 2045.

27. Orth, W. S.; Gillham, R. W.; Environ. Sci. Technol. 1996, 30, 66.

28. Carucci, A.; Manconi, I.; Manigas, L.; Water Sci. Technol. 2007, 55, 209.

29. Martin, J. E.; Herzing, A. A.; Yan, W. L.; Li, X. Q.; Koel, B. E.; Kiely, C. J.; Zhang, W. X.; Langmuir 2008, 24, 4329.

30. Baer, D. R.; Gaspar, D. J.; Nachimuthu, P.; Techane, S. D.; Castner, D. G.; Anal. Bioanal. Chem. 2010, 396, 983.

31. O'Carroll, D.; Sleep, B.; Krol, M.; Boparai, H.; Kocur, C.; Adv. Water Resour. 2013, 51, 104.

32. Wong, M. S.; Alvarez, P. J. J.; Fang, Y.; Akcin, I. N.; Nutt, M. O.; Miller, J. T.; Heck, K. N.; J. Chem. Technol. Biotechnol. 2009, 84, 158.

33. Shi, Z.; Fan, D.; Johnson, R. L.; Tratnyek, P. G.; Nurmi, J. T.; Wu, Y.; Williams, K. H.; J. Contam. Hydrol. 2015, 181, 17.

34. Xue, W.; Huang, D.; Zeng, G.; Wan, J.; Cheng, M.; Zhang, C.; Hu, C.; Li, J.; Chemosphere 2018, 210, 1145.

35. Jiang, D.; Zeng, G.; Huang, D.; Chen, M.; Zhang, C.; Huang, C.; Wan, J.; Environ. Res. 2018, 163, 217.

36. Zhang, T.; Lowry, G. V.; Capiro, N. L.; Chen, J.; Chen, W.; Chen, Y.; Dionysiou, D. D.; Elliott, D. W.; Ghoshal, S.; Hofmann, T.; Hsu-Kim, H.; Hughes, J.; Jiang, C.; Jiang, G.; Jing, C.; Kavanaugh, M.; Li, Q.; Liu, S.; Ma, J.; Pan, B.; Phenrat, T.; Qu, X.; Quan, X.; Saleh, N.; Vikesland, P. J.; Wang, Q.; Westerhoff, P.; Wong, M. S.; Xia, T.; Xing, B.; Yan, B.; Zhang, L.; Zhou, D.; Alvarez, P. J. J.; Environ. Sci.: Nano 2019, 6, 1283. (disponível em https://pubs.rsc.org/en/content/articlehtml/2019/ en/c9en00143c, acessada em Novembro 2019).

37. Reddy, K. R.; Proceedings of the $6^{\text {th }}$ International Congress on Environmental Geotecnics, New Delhi, India, 2010, 163.

38. Schrick, B.; Blough, J. L.; Jones, A. D.; Mallouk, T. E.; Chem. Mater. 2002, 14, 5140

39. He, F.; Zhao, D.; Environ. Sci. Technol. 2005, 39, 3314.

40. Sakulchaicharoen, N.; O'Carroll, D. M.; Herrera, J. E.; J. Contam. Hydrol. 2010, 118, 117

41. Saleh, N.; Phenrat, T.; Sirk, K.; Dufour, B.; Ok, J.; Sarbu, T.;
Matyjaszewski, K.; Tilton, R. D.; Lowry, G. V.; Nano Lett. 2005, 5, 2489.

42. Tiraferri, A.; Chen, K. L.; Sethi, R.; Elimelech, M.; J. Colloid Interface Sci. 2008, 324, 71

43. Cameselle, C.; Reddy, K. R. R.; Darko-Kagya, K.; Khodadoust, A.; ASCE 2013, 139, 23.

44. Phenrat, T.; Liu, Y.; Tilton, R. D.; Lowry, G. V.; Environ Sci. Technol. 2009, 43, 1507 .

45. Petosa, R.; Jaisi, D. P.; Quevedo, I. R.; Elimelech, M.; Tufenkji, N.; Environ. Sci. Technol. 2010, 44, 6532.

46. Batley, G. E.; Kirby, J. K.; McLaughlin, M. J.; Acc. Chem. Res. 2013 , $46,854$.

47. Sun, Y.-P.; Li, X.-q.; Cao, J.; Zhang, W.-X.; Wang, H. P.; Colloids Surf., A 2007, 308, 60.

48. Tosco, T.; Papini, M. P.; Viggi, C. C.; Sethi, R.; J. Cleaner Prod. 2014, $77,10$.

49. Gastone, F.; Tosco, T.; Sethi, R.; J. Colloid Interface Sci. 2014, 421, 33.

50. Dalla Vecchia, E.; Luna, M.; Sethi, R.; Environ. Sci. Technol. 2009, 43, 8942.

51. Bezbaruah, A. N.; Krajangpan, S.; Chisholm, B. J.; Khan, E.; Bermudez, J. J.; J. Hazard. Mater. 2009, 166, 1339.

52. Dong, H.; Lo, I. M. C.; Water Res. 2013, 47, 419.

53. Kanel, S. R.; Manning, B.; Charlet, L.; Choi, H.; J. Nanopart. Res. 2007, 9, 725 .

54. Bennett, P.; He, F.; Zhao, D. Y.; Aiken, B.; Feldman, L.; J. Contam. Hydrol. 2010, 116, 35 .

55. Krol, M. M.; Oleniuk, A. J.; Kocur, C. M.; Sleep, B. E.; Bennett, P.; Xiong, Z.; O'Carroll, D. M.; Environ. Sci. Technol. 2013, 47, 7332.

56. Arshad, M.; Soleymanzadeh, M.; Salvacion, J. W. L.; Salimivahid, F.; J. Colloid Interface Sci. 2014, 426, 241.

57. Quinn, J.; Geiger, C.; Clausen, C.; Brooks, K.; Coon, C.; O’Hara, S.; Krug, T.; Major, D.; Yoon, W. S.; Gavaskar, A.; Holdsworth T.; Environ. Sci. Technol. 2005, 39, 1309 .

58. Berge, N. D.; Ramsburg, C. A.; Environ. Sci. Technol. 2009, 43, 5060.

59. Berge, N. D.; Ramsburg, C. A.; J. Contam. Hydrol. 2010, 118, 105.

60. Zhang, R.; Li, J.; Liu, C.; Shen, J.; Sun, X.; Han, W.; Wang, L.; Colloids Surf., A 2013, 425, 108 .

61. Zhu, H. J.; Jia, Y. F.; Wu, X.; Wang, H.; J. Hazard. Mater. 2009, 172, 1591.

62. Ling, X. F.; Li, J. S.; Zhu, W.; Zhu, Y. Y.; Sun, X. Y.; Shen, J. Y.; Han, W. Q.; Wang, L. J.; Chemosphere 2012, 87, 655.

63. Shu, H. Y.; Chang, M. C.; Chen, C. C.; Chen, P. E.; J. Hazard. Mater 2010, 184,499 .

64. Jiang, Z. M.; Lv, L.; Zhang, W. M.; Du, Q.; Pan, B. C.; Yang, L.; Zhang, Q. X.; Water Res. 2011, 45, 2191.

65. Shi, L. N.; Zhang, X.; Chen, Z. L.; Water Res. 2011, 45, 886.

66. Zhu, H. J.; Jia, Y. F.; Wu, X.; Wang, H.; J. Hazard. Mater. 2009, 172, 1591.

67. Zhang, X.; Lin, S.; Chen, Z. L.; Megharaj, M.; Naidu, R.; Water Res. 2011, 45,3481

68. Su, H.; Fang, Z.; Tsang, P. E.; Fang, J.; Zhao, D.; Environ. Pollut. 2016, 214, 94.

69. Pei, G.; Zhu, Y.; Wen, J.; Pei, Y.; Li, H.; Environ. Pollut. 2020, 256, 113407.

70. Schrick, B.; Hydutsky, B. W.; Blough, J. L.; Mallouk, T. E.; Chem. Mater. 2004, 16, 2187.

71. Tiraferri, A.; Sethi, R.; J. Nanopart. Res. 2009, 11, 635.

72. Johnson, R. L.; Johnson, G. O.; Nurmi, J. T.; Tratnyek, P. G.; Environ. Sci. Technol. 2009, 43, 5455.

73. Reddy, K. R.; Darnault, C.; Darko-Kagya, K.; J. Geotech. Geoenviron. Eng. 2014, 140, 04013013.

74. Saleh, N.; Sirk, K.; Liu, Y.; Phenrat, T.; Dufour, B.; Matyjaszewski, K.; Tilton, R. D.; Lowry, G. V.; Environ. Eng. Sci. 2007, 24, 45. 
75. Hydutsky, B. W.; Mack, E. J.; Beckerman, B. B.; Skluzacek, J. M.; Mallouk, T. E.; Environ. Sci. Technol. 2007, 41, 6418.

76. Reddy, K. R.; Khodadoust, A. P.; Darko-Kagya, K.; J. Hazard., Toxic Radioact. Waste 2012, 16, 68.

77. Reddy, K. R.; Darko-Kagya, K.; Cameselle, C.; Sep. Purif. Technol. 2011, 79, 230.

78. Tosco, T.; Sethi, R.; Environ. Sci. Technol. 2010, 44, 9062.

79. Torkzaban, S.; Wan, J.; Tokunaga, T. K.; Bradford, S. A.; J. Contam. Hydrol. 2012, 136, 86.

80. Yao, K. M.; Habibian, M. T.; O’Melia, C. R.; Environ. Sci. Technol. 1971, 5, 1105 .

81. Hosseini, S. M.; Tosco, T.; Water Res. 2013, 47, 326.

82. Yang, G. C. C.; Tu, H.-C.; Hung, C.-H.; Sep. Purif. Technol. 2007, 58, 166.

83. Kocur, C. M.; Chowdhury, A. I.; Sakulchaicharoen, N.; Boparai, H. K.; Weber, K. P.; Sharma, P.; Krol, M. M.; Austrins, L.; Peace, C.; Sleep, B. E.; O'Carroll, D. M.; Environ. Sci. Technol. 2014, 48, 2862.

84. O'Carroll, D. M. Em Nanotechnology applications for clean water, $2^{\text {nd }}$ ed.; Street, A.; Sustich, R.; Duncan, J.; Savage, N., eds.; William Andrew: Norwich, NY, 2014, p. 441.

85. Hendren, C. O.; Lowry, M.; Grieger, K. D.; Money, E. S.; Johnston, J. M.; Wiesner, M. R.; Beaulieu, S. M.; Environ. Sci. Technol. 2013, 47, 1190.

86. Simunek, J.; Jacques, D.; Van Genuchten, M. Th.; Mallants, D.; J. Am. Water Resour. Assoc. 2006, 42, 1537.

87. Van Genuchten, M. Th.; Simunek, J.; Leij, F. J.; Toride, N.; Sejna, M.; Am. Soc. Agric. Eng. 2012, 55, 1353.

88. Lefevre, E.; Bossa, N.; Wiesner, M. R.; Gunsch, C. K.; Sci. Total Environ. 2016, 565, 889.

89. Paschoalino, M. P.; Marcone, G. P. S.; Jardim, W. F.; Quim. Nova 2010, $33,421$.

90. Brandl, F.; Bertrand, N.; Lima, E. M.; Langer, R.; Nat. Commun. 2015, 6,1 .

91. Danish, M.; Gu, X.; Lu, S.; Naqvi, M.; Environ. Sci. Pollut. Res. Int. 2016, 23, 13298

92. Peralta-Videa, J. R.; Zhao, L.; Lopez-Moreno, M. L.; De La Rosa, G.; Hong, J.; Gardea-Torresdey, J. L.; J. Hazard. Mater. 2011, 186, 1.

93. Lee, C.; Kim, J.; Lee, W.; Nelson, K.; Yoon, J.; Sedlak, D.; Environ. Sci. Technol. 2008, 42, 4927.

94. Li, X.-Q.; Zhang, W.-X.; J. Phys. Chem. 2007, 111, 6939.

95. Crane, R. A.; Scott, T. B.; J. Hazard Mater. 2012, 15, 211.

96. Kanel, S. R.; Manning, B.; Charlet, L.; Choi, H.; J. Nanopart. Res. 2007, 9,725 .

97. Pereira, W. S.; Freire, R. S.; Quim. Nova 2005, 28, 130.
98. Anza, M.; Salazar, O.; Epelde, L.; Alkorta, I.; Garbisu, C.; Front. Environ. Sci. 2019, 7, 19.

99. Cecchin, I.; Tessaro, E. F.; Reginatto, C.; Thome, A.; Reddy, K. R. Em Proceedings of the $8^{\text {th }}$ International Congress on Environmental Geotechnics, vol. 1; Zhan, L.; Chen, Y.; Bouazza, A., eds.; Springer Singapore: Singapore, 2018, p. 882.

100. Riéffel, R.; Paixão, W.; Silva, I. Z.; Ourique, A. F.; Volkmer, T. M.; Sagrillo, M. R. Discip. Sci. 2019, 20, 123.

101. Dong, H.; Li, L.; Lu, Y.; Cheng, Y.; Wang, Y.; Ning, Q.; Wang, B.; Zhang, L.; Zeng, G.; Environ. Int. 2019, 124, 265.

102. Elliott, D. W.; Zhang, W-X.; Environ. Sci. Technol. 2001, 35, 4922.

103. Henn, K. W.; Waddill, D. W.; Remediation J. 2006, 16, 57.

104. He, F.; Zhao, D.; Paul, C.; Water Res. 2010, 44, 2360.

105. Johnson, R. L.; Nurmi, J. T.; Johnson, G. S. O’B.; Fan, D.; Johnson, R. L. O’B.; Shi, Z.; Salter-Blanc, A. J.; Tratnyek, P. G.; Lowry, G. V.; Environ. Sci. Technol. 2013, 47, 1573.

106. Kocur, C. M.; Chowdhury, A. I.; Sakulchaicharoen, N.; Boparai, H. K.; Weber, K. P.; Sharma, P.; Krol, M. M.; Austrins, L.; Peace, C.; Sleep, B. E.; O'Carroll, D. M.; Environ. Sci. Technol. 2014, 48, 2862.

107. Mueller, N. C.; Braun, J.; Bruns, J.; Cerník, M.; Rissing, P.; Rickerby, D.; Nowack, B.; Environ. Sci. Pollut. Res. 2012, 19, 550.

108. Kuiken, T.; Nano Today 2010, 5, 6.

109. Cook, S. M.; Assessing the Use and Application of Zero-Valent Iron Nanoparticle Technology for Remediation at Contaminated Sites; 2009. (disponível em http://www.clu-in.org/download/studentpapers/ZeroValent-Iron-Cook.pdf, acessada em Novembro 2019).

110. Bardos, P.; Bone, B.; Elliott, D.; Hartog, N.; John H.; Paul, N.; A Risk/Benefit Approach to the Application of Iron Nanoparticles for the Remediation of Contaminated Sites in the Environment. Defra Research Project Final Report; 2011. (disponível em http://randd.defra. gov.uk/Default.aspx ?Menu=Menu\&Module=More \&Location=None \& Completed $=0 \&$ ProjectID $=17502$, acessada em Novembro 2019).

111. Christiansen, C. M.; Damgaard, I.; Broholm, M.; Kessler, T.; Klint, K. E.; Nilsson, B.; Bjerg, P. L.; Groundwater Monit. Rem. 2010, 30, 107.

112. Rosansky, S.; Condit, W.; Sirabian, R.; Best Practices for Injection and Distribution of Amendments. Technical Report TR-NAVFAC-EXWCEV-1303, 2013. (disponível em http://www.clu-in.org/remediation/, acessada em Novembro 2019).

113. Wei, Y. T.; Wu, S. C.; Chou, C. M.; Che, C. H.; Tsai, S. M.; Lien, H. L.; Water Res. 2010, 44, 131.

114. https://nanoiron.cz/en/, acessada em Novembro 2019.

115. Cecchin, I.; Reginatto, C.; Thomé, A.; Schnaid, F.; Reddy, K. R.; XVIII Congresso Brasileiro de Mecânica dos Solos e Engenharia Geotécnica COBRAMSEG, Belo Horizonte, Brasil, 2016, 19. 